\title{
Adaptive Fault Tolerant Control for Liquid Tank Process
}

\author{
Mehmet Arıcı ${ }^{1}$, Tolgay Kara ${ }^{2}$
}

Accepted $3^{\text {rd }}$ September 2016

\begin{abstract}
Level control of liquids in coupled tanks is a basic requirement in many industrial processes. Liquid levels in tanks must be controlled accurately regardless of environmental circumstances. Minor faults in sensors, actuators or other system components that take place in processes where liquid level control is required can result in catastrophic consequences. In this case, a fault tolerant control system is needed. The controller must be either robust (passive) or in reconfigurable (active) type in order to compensate for the effect of actuator faults and maintain system reliability and performance. In this study, a water tank level control system and possible valve actuator faults are modelled. By designing different controllers and using modelled failures a simulation is constructed. To test the reconfigurable type controller performance against faults/failures, a model reference adaptive control system is implemented and compared with PI-controlled system.
\end{abstract}

Keywords: Actuator faults, fault modelling, fault tolerant control, model reference adaptive control, liquid tank

\section{Introduction}

Main industries where liquid level and flow control are essential include petro-chemical industries, paper production plants, water treatment industries, food processing industries, pharmaceutical industries, nuclear power generation plants, and automatic water drainage systems. Due to increasing demands on system performance and product quality as well as economic benefits, modern technical processes have become more complicated. These complicated systems are vulnerable to faults in sensors and actuators. Minor faults in sensors, actuators or other system components that take place in processes in which fluid level control is a basic requirement can result in catastrophic consequences. Control system in this case must compensate for the effect of actuator faults and must maintain system reliability and performance.

Failures are difficult to accurately predict in time and can be abrupt, incipient and intermittent [1]. They are classified according to their location of occurrence in the system. The impact of a fault can be a small reduction in efficiency but could also lead to overall system failure and system instability. Therefore, in the design phase of compensators a good actuator failure model that covers all possible cases is crucial and in the application phase, more practical. Different types of actuator models are available in both deterministic and stochastic manners for linear or nonlinear systems. Most of related studies consider deterministic actuator fault models [2]. They use various approaches in deterministic manner [3-15] and generally consider a specific case that once the actuator fails; it will stay at the faulty mode during the rest running process. In other words, the number of actuator failures is finite. However, in practice, especially for safety-critical systems, the failed actuator may recover itself by a self-repairing control system and the actuator may fail more than

\footnotetext{
1, 2 Electrical and Electronics Engineering Department, Engineering Faculty, Gaziantep University, Campus, 27310, Gaziantep/Turkey

* Corresponding Author: Email: mehmetarici@gantep.edu.tr

Note: This paper has been presented at the $3^{\text {rd }}$ International Conference on Advanced Technology \& Sciences (ICAT'16) held in Konya (Turkey), September 01-03, 2016.
}

once during the operation process, that is, the number of actuator failures is infinite. The actuator failures are practically stochastic in nature. For instance, a normally open electrical contact in electrical switches, relays or breakers is required to be open when it is in a de-energized or relaxed state. However, at some moment, the contact may undergo abrupt disturbances, which turns it to be closed, and it may recover to be open again, that is, the contact may jump between closed mode (failure) and open mode (failure-free) in a stochastic manner. Several studies design compensators with stochastic actuator failure [17-25]. In [17-19], actuator failure model output represented with a failure or faulty matrix. This diagonal matrix takes ' 1 ' and ' 0 ' values, which correspond to total failure and normal operation conditions. Also values between ' 1 ' and ' 0 ' are considered which represent loss of effectiveness. The diagonal matrix is stochastic with a known distribution. Generally Bernoulli distributed sequences and Gaussian distribution are used to model failure behaviours. In [20-25], the aforementioned abrupt changes are modelled as a Markovian process. Markovian process is a stochastic model that can be used to model a random system that changes states according to a transition rule that only depends on the current state. [26] shows that for given adequate historical data, the abrupt changes can be modelled as Markovian process. In the studies cited above, the stochastic functions related to Markovian variables are employed to denote the failure scaling factors for each actuator.

It is needed to design control methods capable of ensuring nominal performance considering the occurrence of failures. This control is referred to as fault tolerant control (FTC) which has become of significant importance in the last decades. There are lots of approaches in FTC area and individual research has been carried out extensively. However systematic concepts, design methods and even terminology are still not standardized [2, 3]. In FTC systems, the achievable system performance depends on the availability of redundancies in the control system as well as the design approaches used in synthesis of fault-tolerant controllers. Recently, FTC systems are classified into two categories, namely, active FTCs, and passive FTCs. These two approaches use different design methodologies for the same control objective. 
However, due to the distinctive design approaches used, each method can result in some unique properties. A general requirement for both methods is the existence of system redundancies which can be analytical or applied on hardware and difference is how the redundancy is utilized.

In passive approach, a list of potential malfunctions is assumed to be known a priori as design basis faults, and all failure modes as well as the normal system operating conditions are considered at the design stage. An active FTC reacts to system component malfunctions (including actuators, system itself, and sensors) by reconfiguring the controller based on the real-time information from a Fault Detection Diagnosis (FDD) scheme. The term "active" represents corrective actions taken actively by the reconfiguration mechanism to adapt the control system in response to the detected system faults. Adaptive control methods are very suitable for active FTC. Due to their adaptation ability in the case of system parameter changes, these methods are in selfreconfigurable type. In other words, they do not require the reconfiguration mechanism and FDD components if component and actuator faults are considered.

In this study, firstly, a general model for actuator fault/failure is given. Then, a simple single input single output water level control system with valve actuator is chosen as testbed since it is widely used in process industry. The level control process is modeled and simulated in order to show the effect of actuator faults/failures on closed loop system performance. A conventional PI controller is chosen to track reference water level. Then, in random time and mode, various fault/failure scenarios are taken into account. Conventional PI controller cannot compensate the failure effects since it has not a reconfiguration structure. Therefore a controller with that property is needed. A model reference adaptive controller is designed in this case and fault accommodation property is compared with PI controller.

\section{Problem Formulation and System Descriptions}

\subsection{Actuator Failure Model}

Faults are not a reduction in efficiency only; they also include other cases such as stuck/frozen/hard-over failure of control valves in the case of process control. To represent the actuator faults in a more general formulation, the following mathematical model can be used [26]:

$\left\{\begin{array}{c}u_{a}(t)=\sum_{A} u(t)+\left(\mathrm{I}-\sum_{A}\right) f_{a}(t) \\ \sum_{A}=\operatorname{diag}\left\{\left[\begin{array}{llll}\sigma_{1}^{a}, & \sigma_{2}^{a}, & \ldots, & \sigma_{m}^{a}\end{array}\right]\right\}\end{array}\right.$

where $u_{a}(t)$ is faulty actuator output, $f_{a}(t)$ contains the values at which the actuators are stuck or floating or hard-over. $I$ is the identity matrix and $\sigma_{i}^{a}=0$ represents a total fault (i.e. a complete failure) of the i-th actuator of the system so that the control action coming from this $\mathrm{i}$-th actuator becomes equal to the i-th element of the uncontrollable offset function $f_{a}(t)$, i.e., $u_{a}(t)=f_{a}(t)$. On the other hand, $\sigma_{i}^{a}=1$ implies that $\mathrm{i}$-th actuator operates normally $\left(u_{a}(t)=u(t)\right)$. The quantities $\sigma_{i}^{a}$ can also take values in between 0 and 1 , making it possible to represent partial actuator faults. For different types of fault conditions the above model can be specified in detail as:

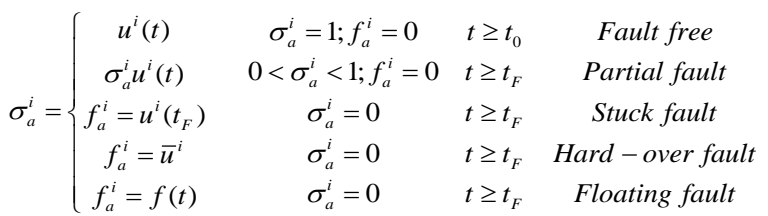

Practically, considered faults in (2) occur randomly with unknown size and mode. Some probabilistic properties can be added to the model to represent randomly occurring phenomenon. In this case, the failure model can also represent the failures occurring intermittently.

Faults are further classified as additive and multiplicative with respect to the way they are modelled. Additive faults are suitable for representing component faults in the system while sensor and actuator faults are mostly multiplicative by nature. Possible actuator faults in multiplicative type can be represented by:

$$
\begin{aligned}
& \dot{x}(t)=A x(t)+B u_{a}(t) \\
& \dot{x}(t)=A x(t)+B \sum_{A}(t) u(t)+B\left(\mathrm{I}-\sum_{A}(t)\right) f_{a}(t) \\
& y(t)=C x(t)
\end{aligned}
$$

Such multiplicative actuator faults do not directly affect the dynamics of the controlled system itself; however they can significantly affect the closed-loop system and may even affect the controllability of the system.

\subsection{System Model}

In water level control system given in fig. 1, water is pumped into the tank at the top through an actuated valve and at the bottom of tank the water drains through a pipe. The objective is to control the water level in the tank with zero steady-state error.

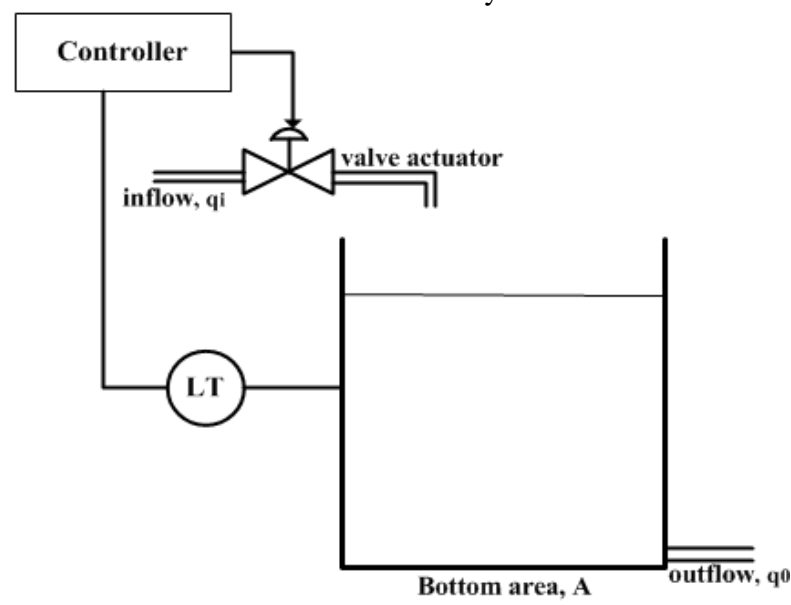

Figure 1. Water level control system

According to Bernoulli equation, the rate of flow of water through the pipe is given by:

$q_{o}=a \sqrt{2 g h}$

where $\mathrm{h}$ is the level of the tank, is the pipe cross-section area and $\mathrm{g}$ is the acceleration of gravity. Conservation of mass yields the equation:

$A \frac{d h}{d t}=q_{i}-q_{o}=q_{i}-a \sqrt{2 g h}$

where $A$ is the bottom area of the tank, $q_{i}$ is the inflow rate through the valve.

If we linearize the system around an operating point and ignore the pressure of the tank outlet, the system can be assumed to be first order but by taking valve dynamics into account, system 
dynamics become second order. The first order system transfer function between inflow rate and water level is:

$\frac{H(s)}{Q_{i}(s)}=\frac{R}{A R s+1}$

where $R=h / q_{o}$ is the resistance at the outlet and the valve dynamics can be given with a first order transfer function between controller output $U(s)$ and system input $M(s)$ :

$G_{v}=\frac{M(s)}{U(s)}=\frac{K_{v}}{T_{v} s+1}$

Here gain $K_{v}$ represents steady-state relation between input and output and $T_{v}$ is the time constant of valve actuator.

\subsection{Fault Tolerant Control Design}

In normal operation conditions, a PI controller is able to track reference while in actuator fault case; an adaptation mechanism is needed because of changing closed loop system parameters. Therefore MRAC is integrated into the system such that PIcontrolled system model in normal operation is the reference model of MRAC. PI controller can be represented by the following transfer function:

$G_{P I}=\frac{U(s)}{E(s)}=K_{p}\left(1+\frac{1}{T_{i} s}\right)$

Reference model is chosen such that valve dynamics and system parameters are unknown. In this case, the closed loop transfer function that has one zero and three poles with unknown coefficients of known signs can be written as follows:

$G_{c l}(s)=\frac{Y(s)}{R(s)}=\frac{b_{1} s+b_{0}}{s^{3}+a_{1} s^{2}+a_{2} s+a_{3}}$

In order to meet MRAC objective with a control law that is free of differentiators and uses only measurable signals, plant and reference model must satisfy some requirements such as plant numerator polynomial is monic, plant degree is known, plant relative degree is known; reference model numerator and denominator are monic Hurwitz polynomials and relative degree is the same as that of plant. In other words, reference model must be chosen to be strictly positive definite (SPR) to obtain a stable controller. Following the certainty equivalence principle, the controller given below can be used [27]:

$u=\theta_{1}^{* T} \frac{\alpha(s)}{\Lambda(s)} u+\theta_{2}^{* T} \frac{\alpha(s)}{\Lambda(s)} y_{p}+\theta_{3}^{* T} y_{p}+c_{0}^{*} r$

where $\alpha(s)=\alpha_{n-2}(s)=\left[s^{n-2}, s^{n-3}, \ldots s, 1\right]$ for $n \geq 2, \alpha(s)=0$ for $n=1$. $\quad \theta_{1}, \theta_{2} \in \mathbb{R}^{n-1} ; \quad c_{0}, \theta_{3}^{*} \in \mathbb{R}$ are constant parameters to be designed and $\Lambda(s)$ is an arbitrary monic Hurwitz polynomial of degree $\mathrm{n}-1$. The controller given in (10) has known parameters ( $\left.\theta^{*}\right)$ i.e., the controller is designed for a known plant transfer function therefore it is named as model reference controller (MRC). However, by using certainty equivalence, we can define an update law and use the same controller with unknown parameters. We can write the equation in compact form as follows:

$u=\theta^{* T} \omega$

Let us define the parameter error as:

$\tilde{\theta}=\theta(t)-\theta^{*} \Rightarrow \theta(t)=\tilde{\theta}+\theta^{*}$

By using (12), (11) can be written as follows:

$u=\tilde{\theta}^{T} \omega+\theta^{* T} \omega$
In order to choose an adaptation law, we have to first find out how the tracking error is related to the parameter error:

$e=G_{m}(s) p^{*}\left(u-\theta^{* T} \omega\right)=G_{m}(s)\left(p^{*} \tilde{\theta}^{T} \omega\right)$

where $G_{m}(s)$ is reference model transfer function; $p^{*}$ is the ratio of the plant and reference model high frequency gain. Following Lyapunov function is used to prove system stability:

$V(\tilde{\theta}, e)=\frac{1}{2} e^{T} P_{m} e+\frac{1}{2}\left|p^{*}\right| \tilde{\theta}^{T} \Gamma^{-1} \tilde{\theta}$

Here $P_{m}=P_{m}^{T}>0$ and $\Gamma=\Gamma^{T}>0$. By using Kalman-Yakubovich lemma and Lyapunov function above, adaptive law is obtained as follows:

$\dot{\theta}=-\operatorname{sgn}\left(\frac{k_{p}}{k_{m}}\right) \Gamma e \omega$

In the design procedure of above controller, we assume that system has relative degree 1 which gives the opportunity to design reference model as SPR. The reference model we consider for the plant has relative degree 2 . In this case reference model $G_{m}(s)$ can no longer be SPR. We can solve this problem by using the fact that $\left(s+p_{0}\right)\left(s+p_{0}\right)^{-1}$ is identity for some $p_{0}>0$ and rewrite the error equation:

$e=G_{m}(s)\left(s+p_{0}\right) p^{*}\left(u_{f}-\theta^{* T} \phi\right)$

with $u_{f}=\frac{1}{s+p_{0}} u, \quad \phi=\frac{1}{s+p_{0}} \omega$, which implies that the control law in compact form is:

$u=\theta^{T} \omega+\dot{\theta}^{T} \phi$

The reference model degree is $\mathrm{n}=3$ hence choosing the degree of $\Lambda(s)$ as $n-1=2, \quad$ fixing $\quad \Lambda(s)=\mathrm{s}^{2}+s+1$ and taking $\alpha(s)=\left[\begin{array}{ll}s & 1\end{array}\right]$ for $n=3$ designed controller takes the following form:

$u=\theta_{1}^{T}(t)\left[\begin{array}{ll}\frac{s}{s^{2}+s+1} & \frac{1}{s^{2}+s+1}\end{array}\right]^{T} u+\theta_{2}{ }^{T}(t)\left[\begin{array}{ll}\frac{s}{s^{2}+s+1} & \frac{1}{s^{2}+s+1}\end{array}\right]^{T} y_{p}$ $+\theta_{3}(t) y_{p}+\theta_{4}(t) r+\dot{\theta}^{T} \phi$

$\dot{\theta}=-\Gamma e \phi \operatorname{sgn}\left(\frac{k_{p}}{k_{m}}\right)$

$\phi=\frac{1}{s+1} \omega$

\section{Simulations}

In this part, firstly, water level control process is modelled and a PI controller is chosen to track reference water level. Using (5), a simulation model is constructed with the model parameters given in table 1.

Table 1. Process Parameters

\begin{tabular}{|l|l|}
\hline Parameter & Value \\
\hline Height of tank, $h$ & $2 \mathrm{~m}$ \\
\hline Bottom area, $A$ & $1 \mathrm{~m}^{2}$ \\
\hline Out pipe cross-section, a & $0.1 \mathrm{~m}^{2}$ \\
\hline Initial level height $h_{i}$ & $0.5 \mathrm{~m}$ \\
\hline
\end{tabular}

Appropriate PI parameters are chosen to achieve good time 
response characteristics such as $10 \%$ maximum overshoot and $10 \mathrm{~s}$ settling time. The parameters that meet the time domain specifications are $\mathrm{Kp}=0.65$ and $\mathrm{Ti}=2.86 \mathrm{~s}$. Fig. 2 shows the simulation diagram of the system with valve dynamics. System simulation time is $150 \mathrm{~s}$ and sample time is selected to be $0.01 \mathrm{~s}$. A square wave reference input that changes between 0.7 and 1.7 is applied and the response of the system in normal mode can be seen in Fig. 3.

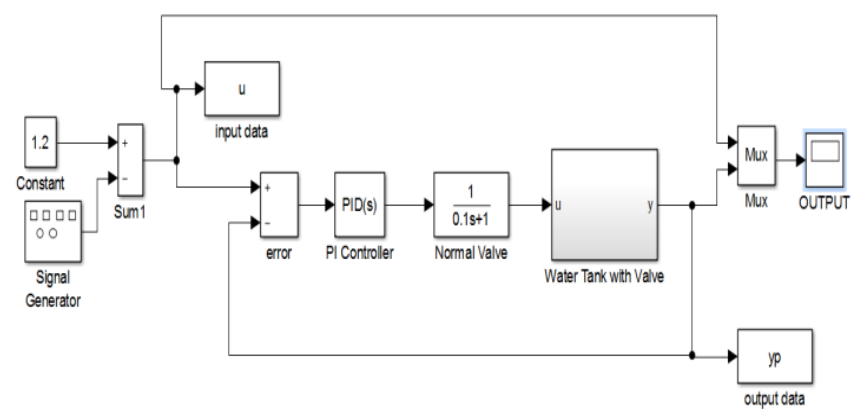

Figure 2. PI-controlled simulated system block structure

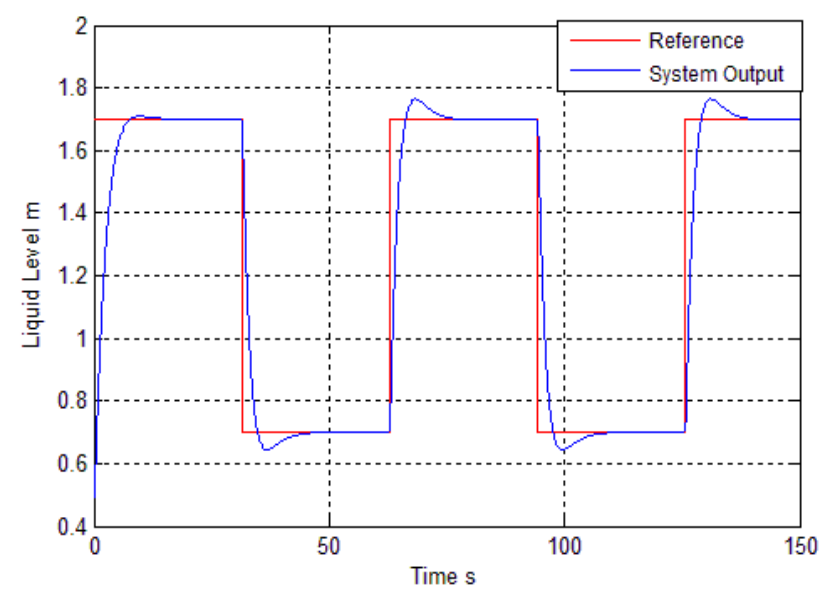

Figure 3. PI-controlled system output response in normal mode

PI controlled healthy system given in Fig. 2 is subjected to least squares system identification process by using reference input data $\mathrm{u}$ and output response data $\mathrm{y}_{\mathrm{p}}$. Plant parameters in the simulation are assumed to be unknown in order to take possible parameter variations and uncertainty in real system into account. As a result of identification the following transfer function is obtained:

$G_{i d}(s)=\frac{Y(s)}{R(s)}=\frac{3.65 s+1.32}{s^{3}+6.70 s^{2}+4.52 s+1.32}$

Fig. 4 shows model reference adaptive controller integrated system simulation model. Before starting the simulations, an actuator failure block is constructed for valve actuator so that in any time of simulation, random failure mode with unknown size can be applied to closed loop system. A wide range of fault/failure cases of valve are considered such as; hard-over, lock-in-place, stuck open, floating failure, leakage, loss of effectiveness. Fig. 5 shows the structure of failure block integrated water tank model. Faulty actuator block with various failure modes that is integrated into simulation can be seen in Fig. 6.

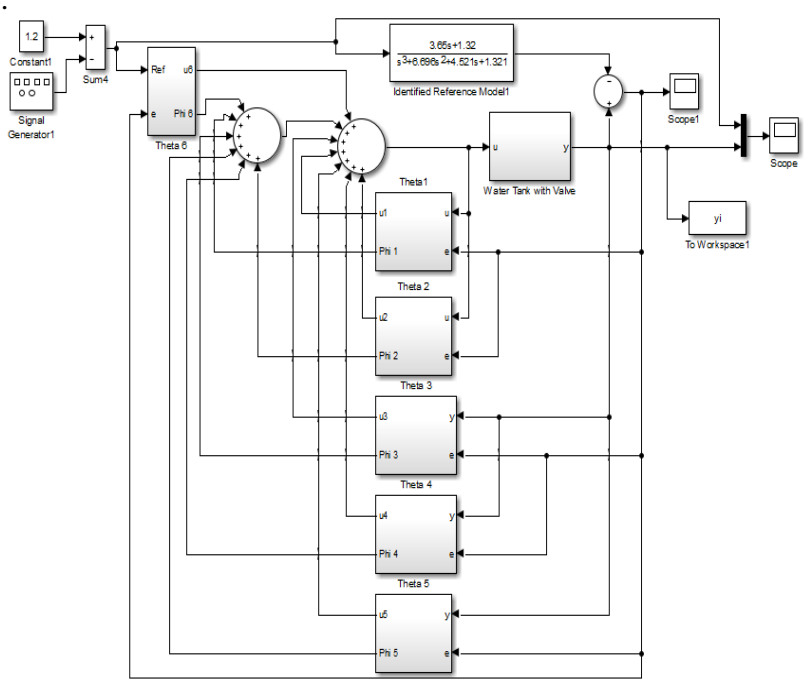

Figure 4. Model reference adaptive controlled system block structure

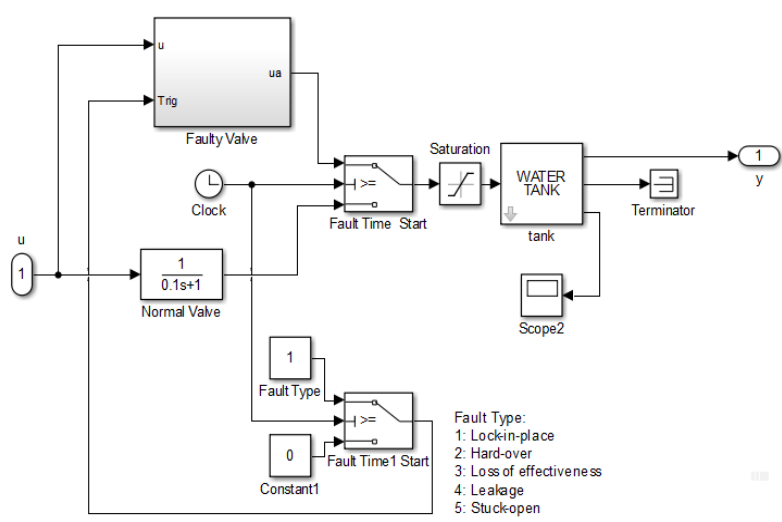

Figure 5. Water tank model with integrated faulty valve block

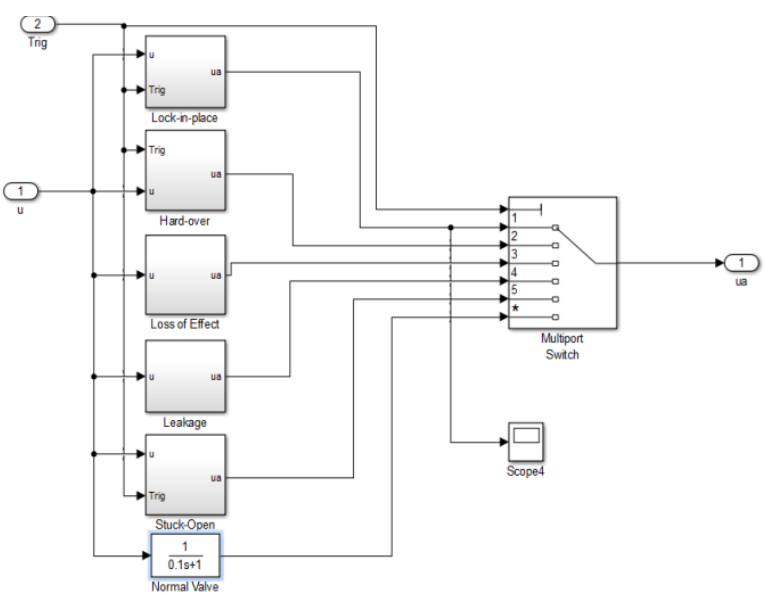

Figure 6. Faulty actuator block with fault/failure modes

In MRAC system simulations, total simulation time is 140 s, sample time is $0.01 \mathrm{~s}$ and a square wave reference input that changes between 0.7-1.7 with a period of $90 \mathrm{~s}$ is applied. Failure responses of both adaptive and non-adaptive controller are examined from different aspects. Various types of failures considered in Section II are simulated. In addition, fault occurrence types that may differ such as incipient, intermittent and abrupt changes are also considered. Following figures show the performance of PI-controlled system and MRAC system in the case of complete or partly failures.

Simulation results show that adaptive controller can compensate for any partial failure regardless of its occurrence type such as 
abrupt, intermittent and incipient. It has also a good transient response while PI-controlled system exhibits undesired high amplitude oscillations and bad transient behavior in the case of intermittent faults. Furthermore, in incipient loss of effectiveness fault mode given in Fig. 7, PI controller may dramatically damage a real process. In abrupt loss of effectiveness case given in Fig. 8, MRAC adapts itself to system changes while PI controller exhibits an undershoot. In Fig. 9, an intermittent valve leakage occurs with 20s duration and PI control has an oscillatory transient behavior. In abrupt valve leakage case which response is given in Fig. 10, PI controlled system output oscillates continuously while MRAC keeps tracking the reference input. In abrupt occurrence type lock-in-place and hard-over failure cases given in Fig. 11 and Fig. 13 respectively, both controller types cannot accommodate the failures since the system has loose controllability property and does not have actuator redundancy. As it can be seen from Fig. 12, after an intermittent lock-in-place failure of $5 \mathrm{~s}$, both systems has the ability to track the reference signal.

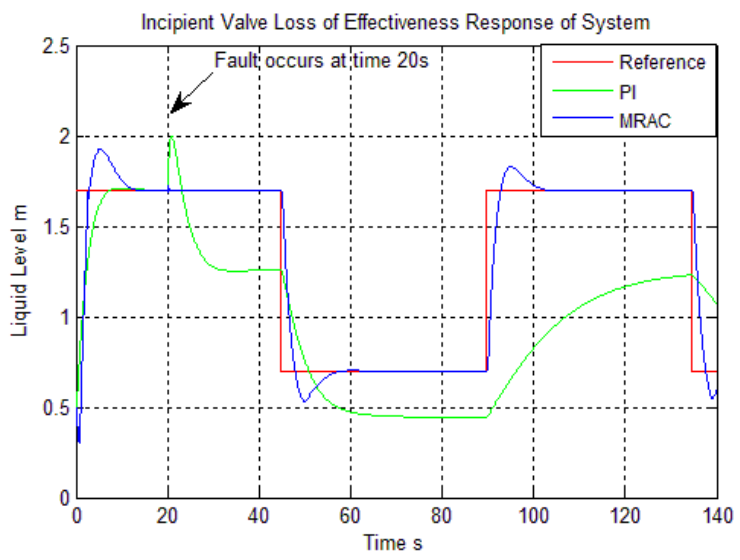

Figure 7. Controlled system responses in incipient leakage mode

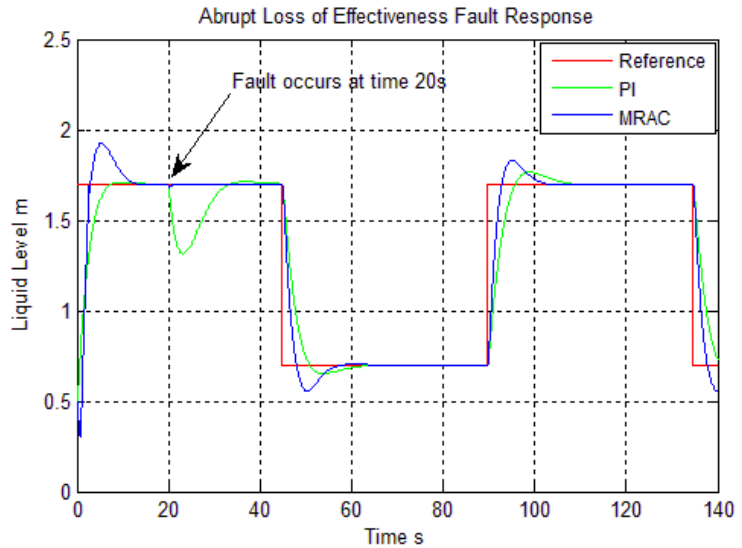

Figure 8. Controlled system response in abrupt loss of effectiveness mode

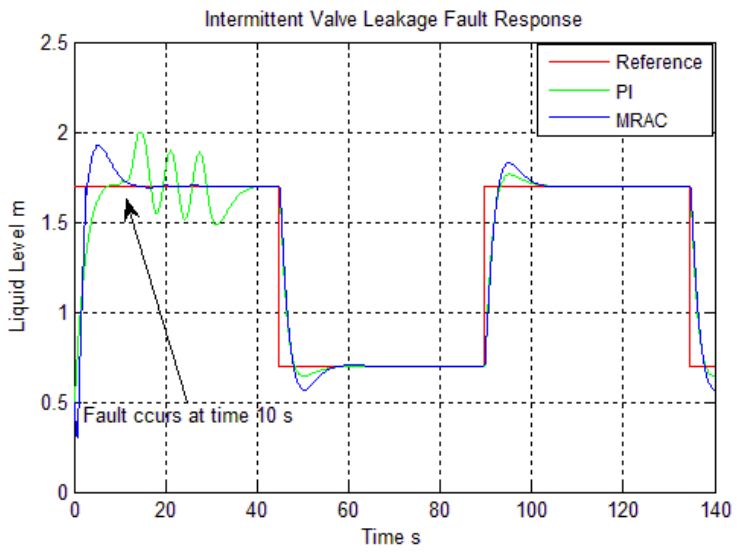

Figure 9. Intermittent leakage failure mode response of controlled system

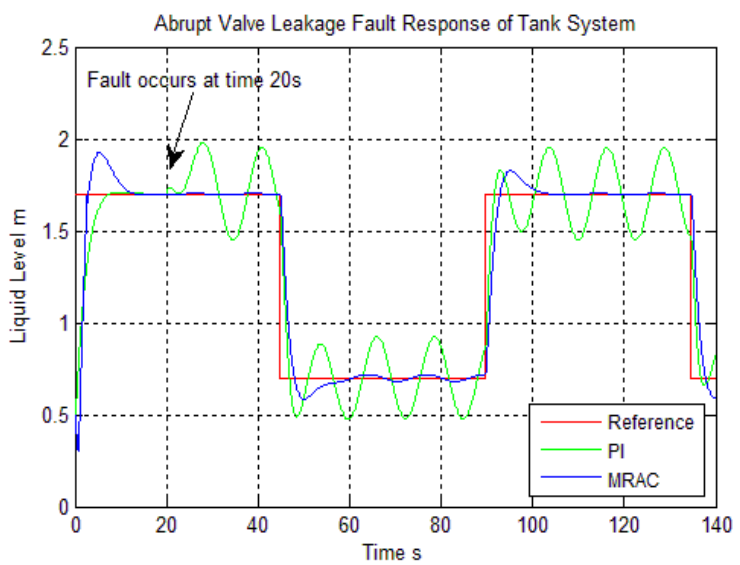

Figure 10. Controlled system response in abrupt leakage fault mode

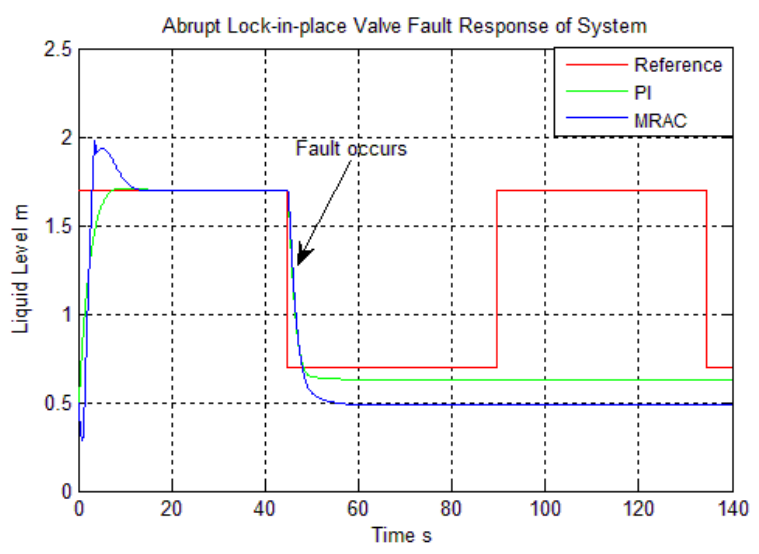

Figure 11. Controlled system response in lock-in-place failure mode

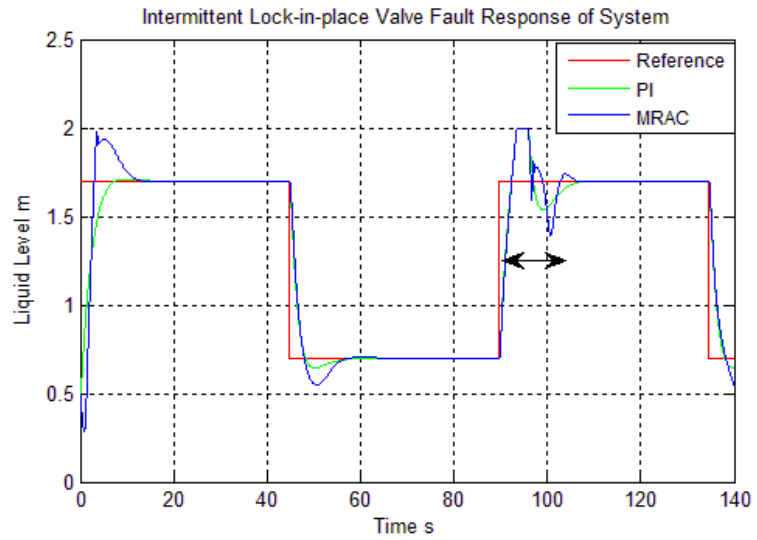

Figure 12. Controlled system response in intermittent lock-in-place mode

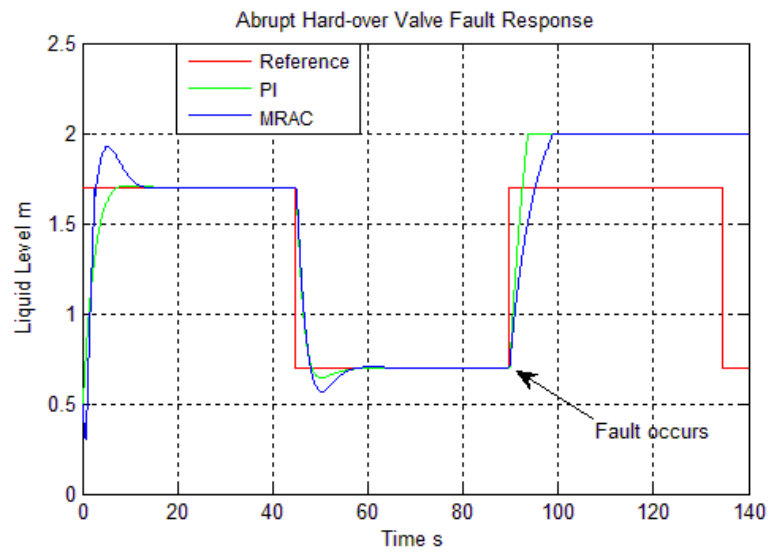

Figure 13. Controlled system response in abrupt hard-over failure mode 


\section{Conclusions}

In this study, a brief literature search about actuator failure modeling is given. A simple water tank level control model, which is widely used in process industry, is selected as benchmark system. Possible valve actuator failures of tank process are considered and modeled. A fault tolerant MRAC controller is designed for the system and controller performance is tested and compared with conventional PI control via simulations. Simulation tests reveal good performance of designed MRAC over conventional control in terms of eliminating actuator failure effects in transient and at the steady state. As a future work, designed controller will be implemented on a real system. Furthermore, stochastic failure model will be integrated into the system at the design stage and also a robust adaptive controller will be designed to compensate for the failures.

\section{Acknowledgement}

This work is supported by The Scientific and Technological Research Council of Turkey (TUBITAK), through project $116 \mathrm{E} 020$.

\section{References}

[1] Y. Mo and B. Sinopoli, "Secure control against replay attacks," in Communication, Control, and Computing, 2009. Allerton 2009. 47th Annual Allerton Conference on, 2009, pp. 911-918.

[2] W. Wang and C. Wen, "Adaptive compensation for infinite number of actuator failures or faults," Automatica, vol. 47, no. 10, pp. 2197-2210, Oct. 2011.

[3] Y. Zhang, "Actuator fault-tolerant control for discrete systems with strong uncertainties," Computers \& Chemical Engineering, vol. 33, no. 11, pp. 1870-1878, Nov. 2009.

[4] X.-Z. Jin and G.-H. Yang, "Robust Adaptive Fault-tolerant Compensation Control with Actuator Failures and Bounded Disturbances," Acta Automatica Sinica, vol. 35, no. 3, pp. 305-309, Mar. 2009.

[5] X.-J. Li and G.-H. Yang, "Robust adaptive fault-tolerant control for uncertain linear systems with actuator failures," IET Control Theory \& Applications, vol. 6, no. 10, pp. 1544-1551, Jul. 2012.

[6] P. Hajiani and J. Poshtan, "Abrupt and incipient fault detection and compensation for a 4-tank system benchmark," Turkish Journal Of Electrical Engineering \& Computer Sciences, vol. 22, pp. 1287-1297, 2014.

[7] Y. Niu, Y. Liu, and T. Jia, "Reliable control of stochastic systems via sliding mode technique: Reliable Control of Stochastic Systems via a Smc Technique," Optimal Control Applications and Methods, vol. 34, no. 6, pp. 712-727, Nov. 2013.

[8] A. Mihankhah, F. R. Salmasi, and K. Salahshoor, "Partial and total actuator faults accommodation for input-affine nonlinear process plants," ISA Transactions, vol. 52, no. 3, pp. 351-357, May 2013.

[9] D. Shin, G. Moon, and Y. Kim, "Design of Reconfigurable Flight Control System Using Adaptive Sliding Mode Control: Actuator Fault," Proceedings of the Institution of Mechanical Engineers, Part G: Journal of Aerospace
Engineering, vol. 219, no. 4, pp. 321-328, Jun. 2005.

[10] J. Jiang and $\mathrm{X}$. Yu, "Fault-tolerant control systems: A comparative study between active and passive approaches," Annual Reviews in Control, vol. 36, no. 1, pp. 60-72, Apr. 2012.

[11] Y. Zhang and J. Jiang, "Bibliographical review on reconfigurable fault-tolerant control systems," Annual Reviews in Control, vol. 32, no. 2, pp. 229-252, Dec. 2008.

[12] P. Millán, L. Orihuela, C. Vivas, F. R. Rubio, D. V. Dimarogonas, and K. H. Johansson, "Sensor-network-based robust distributed control and estimation," Control Engineering Practice, vol. 21, no. 9, pp. 1238-1249, Sep. 2013.

[13] D. Papageorgiou, M. Blanke, H. H. Niemann, and J. H. Richter, "Fault tolerance for industrial actuators in absence of accurate models and hardware redundancy," in Control Applications (CCA), 2015 IEEE Conference on, 2015, pp. 1887-1894.

[14] X.-Z. Jin and G.-H. Yang, "Robust Adaptive Fault-tolerant Compensation Control with Actuator Failures and Bounded Disturbances," Acta Automatica Sinica, vol. 35, no. 3, pp. 305-309, Mar. 2009.

[15] K. H. Johansson, "The quadruple-tank process: a multivariable laboratory process with an adjustable zero," Control Systems Technology, IEEE Transactions on, vol. 8, no. 3, pp. 456-465, 2000.

[16] C. Peng, E. G. Tian, and T. C. Yang, "Robust fault-tolerant control of networked control systems with stochastic actuator failure," IET Control Theory \& Applications, vol. 4, no. 12, pp. 3003-3011, Dec. 2010.

[17] Z. Gu, J. Liu, C. Peng, and E. Tian, "Reliable control for interval time-varying delay systems subjected to actuator saturation and stochastic failure: Reliable Control For Systems With Actuator Saturation \&Amp; Failure," Optimal Control Applications and Methods, vol. 33, no. 6, pp. 739-750, Nov. 2012.

[18] G. Wei, L. Wang, and F. Han, "A gain-scheduled approach to fault-tolerant control for discrete-time stochastic delayed systems with randomly occurring actuator faults," Systems Science \& Control Engineering, vol. 1, no. 1, pp. 82-90, Dec. 2013.

[19] J. Hu, J. Liang, and D. Chen, "Reliable guaranteed-cost control for networked systems with randomly occurring actuator failures and fading performance output," International Journal of General Systems, vol. 44, no. 2, pp. 129-141, Feb. 2015.

[20] M. Mahmoud, J. Jiang, and Y. Zhang, "Optimal control law for fault tolerant control systems," in Decision and Control, 2000. Proceedings of the 39th IEEE Conference on, 2000, vol. 4, pp. 4126-4131.

[21] H. Fan, B. Liu, and Y. Shen, "Fault tolerant control for uncertain systems with actuator stochastic failures," in Control Automation Robotics \& Vision (ICARCV), 2012 12th International Conference on, 2012, pp. 70-75.

[22] B. Chen, Y. Niu, and Y. Zou, "Adaptive sliding mode control for stochastic Markovian jumping systems with actuator degradation," Automatica, vol. 49, no. 6, pp. 1748 1754, Jun. 2013.

[23] H. Fan, B. Liu, W. Wang, and C. Wen, "Adaptive faulttolerant stabilization for nonlinear systems with Markovian jumping actuator failures and stochastic noises," Automatica, vol. 51, pp. 200-209, Jan. 2015.

[24] C. Cheng, Q. Zhao, and F. Tao, "Stability and performance 
of the stochastic fault tolerant control systems," in Decision and Control, 2003. Proceedings. 42nd IEEE Conference on, 2003, vol. 3, pp. 2484-2489.

[25] O.L.V., Costa, M.D., Fragoso, R.P, Marques. "DiscreteTime Markov Jump Linear Systems".Springer, London. 2005.
[26] M., Verhaegen, S., Kanev, R., Hallouzi. "Fault Tolerant Flight Control - A Survey", Lecture Notes in Control and Information Sciences, 2010, vol. 399, pp. 47-89.

[27] P., Ioannou, B. Fidan. "Adaptive Control Tutorial”, Society for Industrial and Applied Mathematics (SIAM). 2006. 